\title{
ALANINO AMINOTRASFERASA EN PANCREATITIS AGUDA DE ORIGEN BILIAR
}

\author{
Guillermo Eduardo Aldana Dimas MD*, Juan David Lleras Riaño MD**, Fanny Acero Fagua MD**, \\ Manuel Guillermo Pabón Parra MD**
}

\begin{abstract}
Resumen
La pancreatitis aguda (PA) es una enfermedad de alta prevalencia e impacto socioeconómico. En nuestro medio la causa principal es la patología biliar. La ultrasonografía endoscópica no siempre está disponible dado su alto costo. Los niveles de alaninoaminotransferasa (ALT) mayores de $150 \mathrm{UI} / \mathrm{l}$ se han relacionado con patología obstructiva de la vía biliar. Objetivo: determinar las características de la ALT en nuestra población para diagnosticar el origen biliar de la PA. Materiales y métodos: estudio de corte transversal en el Hospital de San José de Bogotá DC. Se revisaron historias clínicas con diagnóstico de PA de agosto 2010 a marzo 2012. Se analizaron datos sociodemográficos, clínicos, de laboratorio, imagenológicos, hallazgos intraoperatorios y diagnóstico etiológico. Resultados: se reclutaron 106 pacientes, $67 \%$ mujeres. Se estableció por análisis ROC que los niveles de ALT mayores de 74 UI/ fueron más sensibles y específicos que los de $150 \mathrm{UL} /$. Se encontró la relación de ALT mayor de $74 \mathrm{UI} / \mathrm{l}$ con hiperbilirrubinemia, ictericia y hallazgos ecográficos compatibles con etiología biliar. Conclusión: existe significancia estadística para ALT mayor a $74 \mathrm{UI} / \mathrm{l}$ con etiología biliar (sensibilidad $89 \%$, especificidad $71 \%$ ) con $p<0.001$. Se requieren estudios que comparen esta prueba diagnóstica con ultrasonografía endoscópica.
\end{abstract}

Palabras clave: pancreatitis aguda, pronóstico, coledocolitiasis, colecistectomía, alanino transaminasa (ALT).

Abreviaturas: PA, pancreatitis aguda; ALT, alanino aminotransferasa; CPRE, colangiopancreatografía retrógrada endoscópica.

\section{ALANINE AMINOTRANSFERASE IN BILIARY ACUTE PANCREATITIS}

\begin{abstract}
Acute pancreatitis (AP) exhibits high prevalence and high social and economic impact. It is mainly of biliary etiology in our setting. Endoscopic ultrasonography is not always available due to its high costs. Alanine aminotransferase (ALT) levels greater than $150 \mathrm{UI} / \mathrm{h}$ have been related to biliary duct obstruction. Objective: to determine ALT features among our population to establish biliary etiology. Materials and Methods: a cross sectional study at Hospital de San José, Bogotá DC. The medical records of all patients with AP from August 2010 to March 2012 were reviewed. Social, demographic, clinical, laboratory, imaging, intraoperative findings and etiologic diagnosis data were analyzed. Results: 106 patients were recruited, 67\% females. An ROC analysis established that at ALT levels greater than 74 IU/l there was greater sensibility and specificity than at ALT levels of 150 IU/. The relation between greater than 74 UI// ALT levels with hyperbilirrubinemia, jaundice and ultrasonographic findings compatible with biliary etiology was found. Conclusion: there is statistically significant correlations between ALT levels greater than 74UI/l and biliary etiology (sensibility $89 \%$, specificity $71 \%$ ), $p<0.001$. Further studies comparing this diagnostic test with endoscopic ultrasonography are required.
\end{abstract}

Key words: acute pancreatitis, prognosis, choledocolithiasis, cholecystectomy, alanine transaminase (ALT).

Fecha recibido: octubre 17 de 2012 - Fecha aceptado: marzo 13 de 2013

* Jefe de la Unidad de Trasplantes. Hospital de San José. Profesor Asistente, Fundación Universitaria de Ciencias de la Salud, Bogotá DC. Colombia.
** Residente IV de Cirugía General, Fundación Universitaria de Ciencias de la Salud, Bogotá DC, Colombia. 


\section{Introducción}

La pancreatitis aguda (PA) es una enfermedad de alta prevalencia e impacto socioeconómico con la cual se enfrenta a diario el médico de urgencias y el cirujano general. Es responsable de cerca de 300.000 ingresos hospitalarios y 20.000 muertes al año en EEUU, donde las causas más frecuentes son biliar, idiopática y alcohólica con porcentajes hasta de 54\%, 34\% y $19 \%$. En Colombia la relación etiológica varía con una distribución hasta de $80 \%$ de origen biliar y $9 \%$ alcohólica. ${ }^{1.2}$ Puede ocurrir a cualquier edad pero su mayor frecuencia es entre 30 y 70 años, la cual en ambos sexos es similar y varía según la etiología, siendo mayor en hombres cuando es de origen alcohólico, mientras que la causa biliar es superior en las mujeres. En cuanto a la raza es tres veces más alta su incidencia en los afroamericanos que en la población blanca sin conocer una razón especifica. ${ }^{3,4}$

La PA biliar y alcohólica representan del 70\% al 95\% de los casos, el porcentaje restante está asociado con otras causas como PA posCPRE, hiperlipidemia e hipercalcemia, y de 5 a $15 \%$ corresponde a PA idiopática. Sin embargo, estudios más recientes han descrito que hasta $75 \%$ de las pancreatitis descritas antes como idiopáticas pueden explicarse como de causa biliar secundaria a microlitiasis ${ }^{5}$, no evidente en ecografía transabdominal pero detectable con ultrasonografía endoscópica o en análisis biliar, evidenciando precipitación de cristales de monohidrato de colesterol o gránulos de bilirrubinato de calcio..$^{2.46-8}$ Aunque la litiasis biliar es la principal causa de PA a nivel mundial, solo de 1 a $8 \%$ de los pacientes con cálculos biliares desarrollan esta enfermedad. ${ }^{2}$

Dentro del enfoque inicial de los pacientes con PA es importante identificar la etiología de la misma y así se puede determinar el manejo según la causa especifica. ${ }^{9}$ Teniendo en cuenta lo anterior, es necesario establecer si la pancreatitis es de origen biliar, ya que en los casos severos los pacientes se benefician de CPRE con extracción de cálculos y en los casos leves se requiere la realización de colecistectomía una vez resuelto el episodio de pancreatitis. ${ }^{\mathbf{1 0}-12}$ Antes es necesario descartar o resolver la presencia de cálculos en la vía biliar, con el fin de evitar las recurrencias que ocurren entre $10 \%$ y $63 \% \%^{9.11 .13}$, lo que confiere mayor morbimortalidad al paciente en cada episodio. ${ }^{6.14,15}$

La ecografía hepatobiliar es el examen inicial para el estudio de la etiología de la PA.Las pruebas de función hepática son útiles para predecirsi la PA puede ser secundaria a patología biliar cuando cursa con hiperbilirrubinemia a expensas de la directa y elevación de la fosfatasa alcalina y las transaminasas ${ }^{7.16,17} \mathrm{La}$ ALT mayor a $150 \mathrm{U} / \mathrm{l}$ tiene valor predictivo positivo mayor de $95 \%$ para PA de origen biliar, con rangos entre $78 y$ $100 \%$ en los diferentes estudios ${ }^{18}$, siendo esta la más útil para el diagnóstico entre las pruebas bioquímicas e inclusive algunos autores sugieren que puede remplazar la endoscopia abdominal para determinar la etiología biliar. ${ }^{6-14}$

Debido a la alta frecuencia de esta patología en nuestra institución, decidimos realizar un estudio donde se puedandeterminar las características operativas de la ALT para diagnosticar el origen biliar de la PA yrelacionarla con las otras pruebas diagnósticas utilizadas de rutina en estos pacientes.

\section{Métodos}

Se realizó un estudio de corte transversal con todos los pacientes que acudieron al Hospital de San José de Bogotá DC con diagnóstico de PA por clínica y confirmado por elevación de la amilasa al momento del ingreso tres veces por encima de su valor normal, en el período comprendido entre el 1 de agosto 2010 y el 1 de marzo 2012. Se recogieron los datos concernientes al estudio de su historia clínica. Se excluyeron las embarazadas y aquellos con falla renal crónica ya que en ellos el comportamiento de los marcadores séricos es diferente, así como los que cursaban con pancreatitis crónica y agudización de la misma. Los pacientes con remisión extrainstitucional que no tuvieron estudios completos también salieron del estudio.

Se recolectaron datos demográficos (edad y género), clínicos (presencia de ictericia, coluria y acolia), laboratorio ALT aspartato aminotrasferasa (AST), fosfatasa alcalina (FA) y bilirrubinas (total, directa 
e indirecta), imagenológicos (hallazgos de ecografía hepatobiliar, colangiorresonancia nuclear magnética o tomografía axial computarizada y CPRE), datos intraoperatorios (hallazgos quirúrgicos y de la colangiografía intraoperatoria) y los del diagnóstico final (pancreatitis biliar, alcohólica, posCPRE e idiopática).

Se realizó un análisis de tipo descriptivo. Las variables cualitativas se resumieron con frecuencias absolutas y porcentajes, y las cuantitativas con medidas de tendencia central y de dispersión. Se exploró la asociación entre niveles de ALT y la etiología de la PA haciendo uso de la prueba estadística $\mathrm{X}^{2}$ con niveles de ALT en U/l menores de 74 a 149 y mayores de 150 . Para describir la relación entre los niveles de ALT y la etiología, los hallazgos y las variables sociodemográficas y clínicas, se realizó un análisis de correspondencias múltiples, tomando como variable ilustrativa los niveles de ALT menores de 74 U/l, de 74 a 149 y mayores de 150 , y como variables activas la etiología, los hallazgos y las variables sociodemográficas y clínicas. Se hizo un análisis ROC para establecer los niveles de ALT con mayor sensibilidad y especificidad para el diagnóstico de PA de origen biliar. El análisis estadístico de la información se realizó en Stata 10 y Spad 7.

\section{Resultados}

Se revisaron las historias clínicas de 160 pacientes con diagnóstico de PA distribuidos en rangos de edad, 30 menores de 30 años, 32 entre 30 y 50, y 40 casos mayores de 50 años (Tabla 1). La PA se presentó en mujeres con una prevalencia de 71/106. En cuanto al diagnóstico final la PA se estableció como de origen biliar en $74.5 \%$, alcohólica 3\%, posterior a CPRE $2 \%$ e idiopática $21 \%$ (Tabla 2). Las características clínicas clásicas de obstrucción de la vía biliar (ictericia, coluria y acolia) solo se presentaron en $32 \%$ de los casos; la hiperbilirrubinemia en 41 de los cuales 34 fue directa.
En cuanto a los hallazgos imagenológicos, la ecografía hepatobiliar reportó hallazgos positivos en $73 \%$ de los pacientes, de los cuales el $60 \%$ presentaban colelitiasis y $23 \%$ hallazgos compatibles con colédocolitiasis. El $20 \%$ de los pacientes a quienes se les realizó TAC o resonancia nuclear magnética $(\mathrm{n}=24)$ mostraron hallazgos sugestivos de etiología biliar. El $47 \%$ de los casos fueron llevados a CPRE y de estos $66 \%$ presentaron hallazgos probables de colédocolitiasis y $26 \%$ barro biliar. Fueron llevados a algún procedimiento quirúrgico el $56 \%$, siendo la colecistectomía por laparoscopia la más frecuente $(89.2 \%)$.

\begin{tabular}{|c|c|}
\hline Característica & $\begin{array}{l}\text { No. (\%) } \\
N=106\end{array}$ \\
\hline $\begin{array}{l}\text { Edad (años), mediana (rango)* } \\
<30 \\
30-50 \\
>50\end{array}$ & $\begin{array}{c}44(28-59) \\
30(28) \\
32(30) \\
44(42)\end{array}$ \\
\hline $\begin{array}{l}\text { Género } \\
\text { masculino } \\
\text { femenino }\end{array}$ & $\begin{array}{l}35(33) \\
71(67)\end{array}$ \\
\hline Ictericia & $34(32)$ \\
\hline Coluria & $19(18)$ \\
\hline Acolia & $7(7)$ \\
\hline $\begin{array}{l}\text { ALT } \\
>74 \text { UIII }(n=97) \\
>150 \text { UI/I }(n=97) \\
>300 \text { UIII }(n=97)\end{array}$ & $\begin{array}{l}72(74) \\
60(62) \\
39(40)\end{array}$ \\
\hline AST elevada $(n=98)$ & $79(80)$ \\
\hline FA $>126 \mathrm{mg} / \mathrm{dl} \quad(\mathrm{n}=99)$ & $68(69)$ \\
\hline $\begin{array}{l}\text { Hiperbilirrubinemia }{ }^{\dagger} \\
\text { directa }(B D>50 \% \text { de } B T) \\
\text { indirecta }(B \mid>85 \% \text { de } B T) \\
\text { mixta }\end{array}$ & $\begin{array}{c}41(42) \\
34(83)^{\ddagger} \\
0(0)^{\ddagger} \\
7(17)^{\ddagger}\end{array}$ \\
\hline
\end{tabular}

Los datos presentados son frecuencias absolutas y porcentajes a menos que se indique lo contrario. ${ }^{*}$ Rango interquartílico; $†$ bilirrubina total $\geq 2$; $\neq n=41$; ALT: alanino-aminotransferasa; AST: aspartatoaminotransferasa; FA: fosfatasa alcalina; BT: bilirrubinas totales; BD: bilirrubina directa; BI: bilirrubina indirecta.

\begin{tabular}{|c|c|c|c|c|c|}
\hline \multicolumn{7}{|c|}{ Tabla 2. Distribución etiológica de la pancreatitis aguda } \\
\hline Etiología & Biliar (\%) & Alcohólica (\%) & PostCPRE (\%) & Idiopática (\%) & Total \\
\hline N (\%) & $79(75)$ & $3(3)$ & $2(2)$ & $22(21)$ & 106 \\
\hline
\end{tabular}


Se realizó curva de ROC (Tabla 3) identificando 74 UI/l como el nivel de ALT con mayor sensibilidad y especificidad con cifras de $89 \%$ y $70.8 \%$, con un valor predictivo positivo de $90.3 \%$ y negativo de $68 \%$. Los niveles de $150 \mathrm{UI} / \mathrm{l}$ muestran en nuestro estudio una sensibilidad de $74 \%$ y especificidad de $75 \%$, con valor predictivo positivo de $90 \%$ y negativo de $48.6 \%$. Con $300 \mathrm{UI} / 1$ la sensibilidad fue de $48 \%$, especificidad de $83.3 \%$, valor predictivo positivo $89.7 \%$ y negativo 34.5 .

La ALT menor de 74 UI/l se presentó en 26\%; entre 74 y 149 en $74 \%$ y mayor de 150 en $61 \%$. Se encontró una relación estadística significativa $($ con $\mathrm{p}<0.05)$ entre niveles de ALT mayores de $74 \mathrm{UI} / \mathrm{l}$ con la hiperbilirrubinemia, ictericia clínica y hallazgos compatibles con etiología biliar por ecografía (Tabla 4).

Se detectó relación directa entre etiología biliar y ALT por encima de $74 \mathrm{UI} / 1$ con $\mathrm{p}<0.001$, presentándose en $89 \%$ de los pacientes con PA de origen biliar y en $30 \%$ de los idiopáticos (Tabla 5).

\section{Discusión}

La PA es una enfermedad de alto impacto a nivel mundial. Representa un reto para el ejercicio diario del cirujano, quien debe establecer la etiopatogenia para definir el manejo específico para cada caso. Su factor

Tabla 3. Curva ROC para niveles de ALT en relación con etiología biliar en PA

\begin{tabular}{|c|c|c|}
\hline $\begin{array}{c}\text { Nivel de ALT } \\
\text { (UI/I) }\end{array}$ & Sensibilidad & Especificidad \\
\hline$>50$ & $90.41 \%$ & $45.83 \%$ \\
$>\mathbf{7 4}$ & $89.04 \%$ & $70.83 \%$ \\
$>$ 100 & $80.82 \%$ & $70.83 \%$ \\
$>$ 150 & $75.34 \%$ & $75.00 \%$ \\
$>$ 200 & $65.75 \%$ & $75.00 \%$ \\
$>$ 300 & $47.95 \%$ & $79.17 \%$ \\
$>$ 400 & $42.47 \%$ & $83.33 \%$ \\
$>\mathbf{5 0 0}$ & $35.62 \%$ & $95.83 \%$ \\
\hline
\end{tabular}

\begin{tabular}{|c|c|c|c|}
\hline Caracteristica & $\begin{array}{l}\text { ALT } \\
<74\end{array}$ & $\underset{>=74}{\text { ALT }}$ & $\begin{array}{l}\text { ALT } \\
>=150\end{array}$ \\
\hline \multicolumn{4}{|l|}{ Edad $(N=97)$} \\
\hline$<30$ & 10 & 15 & 14 \\
\hline $30-50$ & 4 & 26 & 21 \\
\hline$>50$ & 11 & 31 & 25 \\
\hline \multicolumn{4}{|l|}{ Tipo de hiperbilirrubinemia* $(\mathrm{N}=93)$} \\
\hline no & 20 & 32 & 26 \\
\hline mixta & 0 & 7 & 7 \\
\hline expensas de la bilirrubina directa & 3 & 31 & 26 \\
\hline \multicolumn{4}{|l|}{ Hallazgos ecográficos* $(\mathrm{N}=96)$} \\
\hline no biliar & 15 & 12 & 11 \\
\hline colelitiasis & 3 & 37 & 30 \\
\hline colédocolitiasis & 2 & 14 & 11 \\
\hline barro biliar & 1 & 1 & 1 \\
\hline microlitiasis & 1 & 3 & 2 \\
\hline poscolecistectomía & 2 & 5 & 5 \\
\hline \multicolumn{4}{|l|}{ Hallazgos en CPRE ( $N=43)$} \\
\hline via biliar normal & 0 & 1 & 0 \\
\hline microlitiasis & 0 & 6 & 6 \\
\hline barro biliar & 1 & 10 & 8 \\
\hline colédocolitiasis & 2 & 20 & 17 \\
\hline CPRE fallida & 0 & 3 & 3 \\
\hline $\begin{array}{l}\text { Procedimiento durante CPRE (N=42) } \\
\text { ninguna } \\
\text { papilotomía } \\
\text { papilotomía y extracción de cálculos }\end{array}$ & $\begin{array}{l}0 \\
0 \\
2\end{array}$ & $\begin{array}{c}3 \\
6 \\
31\end{array}$ & $\begin{array}{c}2 \\
4 \\
28\end{array}$ \\
\hline \multicolumn{4}{|l|}{ Procedimiento quirúrgico $(\mathrm{N}=97)^{*}$} \\
\hline no quirúrgico & 19 & 24 & 22 \\
\hline colecistectomía por laparoscopia & 5 & 44 & 35 \\
\hline colecistectomía abierta & 0 & 2 & 1 \\
\hline $\begin{array}{l}\text { colecistectomía con exploración de } \\
\text { vías biliares }\end{array}$ & 0 & 2 & 2 \\
\hline necrosectomía & 1 & 0 & 0 \\
\hline \multicolumn{4}{|l|}{ Hallazgos intraoperatorios ( $N=54)$} \\
\hline barro biliar & 1 & 8 & 6 \\
\hline colelitiasis sin colecistitis & 2 & 20 & 19 \\
\hline colelitiasis con colecistitis & 1 & 18 & 12 \\
\hline colecistocolédocolitiasis & 1 & 1 & 1 \\
\hline \multicolumn{4}{|c|}{ Hallazgos en colangiografía intraoperatoria $(N=18)$} \\
\hline normal & 2 & 12 & 11 \\
\hline defectos de llenado de vía biliar & 1 & 0 & 0 \\
\hline dilatación de vía biliar & 0 & 2 & 1 \\
\hline $\begin{array}{l}\text { defectos de llenado y dilatación de } \\
\text { vía biliar }\end{array}$ & 0 & 1 & 0 \\
\hline
\end{tabular}

$* p>0.05$ 


\begin{tabular}{|l|c|c|c|c|}
\hline \multicolumn{5}{|c|}{ Tabla 5. Correlación entre etiología de la PA y niveles de ALT } \\
\hline ALT & $\begin{array}{c}\text { Biliar } \\
(\%)\end{array}$ & $\begin{array}{c}\text { Alcohólica } \\
(\%)\end{array}$ & $\begin{array}{c}\text { PosCPRE } \\
(\%)\end{array}$ & $\begin{array}{c}\text { Idiopática } \\
(\%)\end{array}$ \\
\hline$<74$ UIII & $8(11)$ & $2(66)$ & $0(0)$ & $15(75)$ \\
\hline$>75-149$ UI/I & $65 *(89)$ & $1(33)$ & $1(100)$ & $5(25)$ \\
\hline$>150 \mathrm{UI} / \mathrm{I}$ & $54 \dagger(73)$ & $0(0)$ & $\mathrm{I}(100)$ & $5(2)$ \\
\hline Total (n 97) & $73(75)$ & $3(3)$ & $\mathrm{I}(1 \%)$ & $20(21 \%)$ \\
\hline
\end{tabular}

ALT: = alanino-aminotransferasa; $*$ p $<0.001 ; \dagger \mathrm{p} \leq 0,05$.

etiológico principal es la patología biliar representando entre 70 y $80 \%$ en nuestro medio.

La ecografía transabdominal se debe realizar en forma rutinaria en el estudio de la PA tanto para determinar hallazgos inflamatorios del páncreas y estructuras vecinas, como para identificar o descartar la etiología biliar de la misma. ${ }^{19}$ La presencia de proceso inflamatorio del páncreas puede verse hasta en $90 \%$ de los casos. ${ }^{20}$ Este examen es muy útil para el diagnóstico de colelitiasis con sensibilidad entre el 90 y $95 \%$ en los casos no complicados; en distensión de asas por íleo la sensibilidad cae entre 67 y $87 \%^{21}$, con especificidad de $92 \%$, valor predictivo positivo del $100 \%$ y negativo de $75 \%$. Sin embargo, su sensibilidad para la detección de cálculos en el conducto biliar es baja, de 20 a $50 \%$. ${ }^{6,14,22-24}$

El estándar de oro para el diagnóstico de litiasis en la vía biliar es la ultrasonografía endoscópica (UE) con sensibilidad entre $91 \%$ y $100 \%$, especificidad entre $96 \%$ y $99 \%$, valor predictivo positivo hasta $99 \%$ y negativo hasta $98 \% .{ }^{14} \mathrm{La}$ utilización de la UE ha incrementado la posibilidad de diagnóstico de PA de origen biliar en especial para el estudio de los pacientes con PA idiopáticas, de los cuales hasta $75 \%$ pueden deberse a patología biliar no csclarccida por otros estudios diagnósticos. ${ }^{5}$ Es más sensible que la ecografía transabdominal para la identificación de colelitiasis y colédocolitiasis. ${ }^{23}$ A pesar de considerarse este estudio como definitivo para el diagnóstico, algunos autores recomiendan la CPRE más que la UE, ya que la primera se considera superior como costo efectiva, puesto que además de diagnóstica en un buen porcentaje de los casos también logra ser terapéutica y se reservaría la UE para casos que impliquen un mayor reto diagnóstico. ${ }^{25}$
A pesar de lo anterior, la UE no es de uso frecuente en nuestro medio dado su alto costo y la baja disponibilidad, siendo esto una limitante para su uso. De allí surge la necesidad de buscar otras estrategias. En el presente estudio se ha evaluado la utilidad de las pruebas de función hepática como predictoras del origen biliar en PA.

Aunque la ecografía es un medio diagnóstico de gran utilidad para definir la etiología biliar su especificidad es cercana al 90\%, sin embargo en combinación con niveles de ALT mayores de $150 \mathrm{U} / 1$ su especificidad sube a $100 \%$.Un estudio realizado en Nueva Zelanda $^{14}$ en el que se compararon los hallazgos ecográficos con los niveles de ALT, encontró que cerca de $100 \%$ de los pacientes en quienes se documentaba patología biliar asociada con PA tenían elevación de ALT mayor de $150 \mathrm{U} / 1^{24}$, y que aquellos con ecografía negativa para patología biliar podrían presentarla entre 21 y $80 \%$ documentándola por los niveles de ALT, lográndose una reducción de las tasas de subdiagnóstico del origen biliar y disminuyendo de esta forma las recurrencias. ${ }^{6,22,26} \mathrm{Algunos}$ estudios han encontrado como factores predictores independientes de PA de origen biliar el género femenino, edad mayor de 50 años (sensibilidad de $73 \%$ y especificidad de $65 \%$ ) y los niveles ya mencionados de ALT, con una sensibilidad de $93 \%$ y especificidad de $85 \%$ al utilizar los tres factores juntos. En el estudio de Lévy y Boruchowicz se describe el cálculo de la siguiente ecuación como una ayuda para la predicción diagnóstica de PA de origen biliar así:

\section{1}

$1+\exp [(4.6967)-(0,0656 \times$ edad $)+(1.1208 \times$ género $)-(0.6909 \times$ alt $)]$ 
( 1 =hombre, $0=$ mujer, ALT expresado en número de veces por encima del valor normal). ${ }^{6,15,27,28} \mathrm{Al}$ gunos estudios también reportan que hasta el $20 \%$ de las PA pueden presentar pruebas de función hepática normales y no existe claridad en la literatura del comportamiento de la ALT en pancreatitis de etiología distinta a la biliar. ${ }^{29}$ En nuestro estudio se demostró que la relación de patología biliar y elevación de la ALT mayor de 74 UI/l fue más sensible y específica en nuestra población en comparación con lo descrito en la literatura con valores mayores a $150 \mathrm{UI} / \mathrm{l}$.

Recientes estudios han reportado que una ecografía negativa para cálculos biliares asociada con niveles normales de ALT reducen la probabilidad de tener patología biliar en el contexto de una PA hasta un $4 \%$. Esta combinación mejora la sensibilidad y especificidad de ambos estudios a $91 \%$ y $100 \%$ con valor predictivo positivo de $100 \%$ y negativo de $96 \%$. $^{6,14}$

En el análisis de los datos de nuestro estudio se encontró significancia estadística para los valores de ALT mayores de 74UI/l asociados con otros predictores clínicos como ictericia y niveles elevados de bilirrubinas, así como su relación con los hallazgos ecográficos sugestivos de origen biliar, permitiéndonos concluir que esta prueba diagnóstica es de utilidad en el momento de establecer la etiología de la PA y así definir una conducta quirúrgica, en cuyo caso sería colecistectomía laparoscópica en la mayoría de los casos. Se hace necesario complementar con estudios que comparen estos resultados con los de la ultrasonografía endoscópica.

\section{Referencias}

1. Uhl W, Muller CA, Krahenbuhl L, Schmid SW, Scholzel S, Buchler MW. Acute gallstone pancreatitis: timing of laparoscopic cholecystectomy in mild and severe diseasc. Surg Endosc. 1999 Nov;(11):1070-6.

2. Nieto J, Rodriguez S. Manejo de la pancreatitis aguda: guía de práctica clínica basada en la mejor información disponible. Rev Colomb Cir. 2010;25:76-96

3. Stabuc B, Drobne D, Ferkolj I, Gruden A, Jereb J. Kolar G, et al. Acute biliary pancreatitis: detection of common bile duct stones with endoscopic ultrasound. Eur J Gastroenterol Hepatol. 2008 Dec;20(12):1171-5.

4. Cappell MS. Acute pancreatitis: etiology, clinical presentation. diagnosis, and therapy. Med Clin N Am. 2008 Jul;(4):889-x.

5. Saraswat VA, Sharma BC. Agarwal DK, Kumar R, Negi TS, Tandon RK. Biliary microlithiasis in patients with idiopathic acute pancreatitis and unexplained biliary pain: response to therapy. J Gastroenterol Hepatol. 2004 Oct;19(10):1206-11.
6. Kaw M. Brodmerkel GJ, Jr. ERCP, biliary crystal analysis, and sphincter of Oddi manometry in idiopathic recurrent pancreatitis. Gastrointest Endosc. 2002 Feb;55(2):157-62

7. Anderson K, Brown LA, Daniel P, Connor SJ. Alanine transaminase rather than abdominal ultrasound alone is an important investigation to justify cholecystectomy in patients presenting with acute pancreatitis. HPB (Oxford). 2010 Jun; 12(5):342-7.

8. Alexakis N, Lombard M, Raraty M. Ghaneh P, Smart HL, Gilmore I, et al. When is pancreatitis considered to be of biliary origin and what are the implications for management?. Pancreatology. 2007: 7(2-3):131-41.

9. Kiriyama S. Gabata T. Takada T. Hirata K, Yoshida M, Mayumi T. et al. New diagnostic criteria of acute pancreatitis. J Hepatobiliary Pancreat Sci. 2010 Jan;(1):24-36.

10. Kalloo AN, Kantsevoy SV. Gallstones and biliary disease. Prim Care. 2001 Sep:28(3):591-606

11. Chak A. Hawes RH, Cooper GS, Hoffman B, Catalan• MF. Wong RC, et al. Prospective assessment of the utility of EUS in the evaluation of gallstone pancreatitis. Gastrointest Endosc. 1999 May;(5):599-604.

12. Ainsworth AP, Rafaelsen SR, Wamberg PA, Pless T, Durup J, Mortensen MB. Cost-effectiveness of endoscopic ultrasonography, magnetic resonance cholangiopancreatography and endoscopic retrograde cholangiopancreatography in patients suspected of pancreaticobiliary disease. Scand J Gastroenterol. 2004 Jun:(6):579-83.

13. Smotkin J, Tenner S. Laboratory diagnostic tests in acute pancreatitis. J Clin Gastroenterol. 2002 Apr; (4):459-62.

14. Liu CL. Fan ST, Lo CM, Tso WK, Wong Y, Poon RT, et al. Clinico-biochemical prediction of biliary cause of acute pancreatitis in the era of endoscopic ultrasonography. Aliment Pharmacol Ther. 2005 Sep 1:(5):423-31.

15. Levy P, Boruchowicz A, Hastier P. Pariente A. Thevenot T. Frossard JL. et al. Diagnostic criteria in predicting a biliary origin of acute pancreatitis in the era of endoscopic ultrasound: multicentre prospective evaluation of 213 patients. Pancreatology. 2005;(4-5):450-6.

16. Dholakia K, Pitchumoni CS, Agarwal N. How often are liver function tests normal in acute biliary pancreatitis?. J Clin Gastroenterol. 2004 Jan; (1):81-3.

17. Raraty MG, Finch M, Neoptolemos JP. Acute cholangitis and pancreatitis secondary to common duct stones: management update. World J Surg. 1998 Nov;(II):1155-61.

18. Wang SS, Lin XZ, Tsai YT, Lee SD, Pan HB, Chou YH, et al. Clinical significance of ultrasonography, computed tomography, and biochemical tests in the rapid diagnosis of gallstone-related pancreatitis: a prospective study. Pancreas. 1988:(2): 153-8

19. Chwistek M, Roberts I, Amoateng-Adjepong Y. Gallstone pancreatitis: a community teaching hospital experience. J Clin Gastroenterol. 2001 Jul;(I):41-4.

20. Alexakis N, Neoptolemos JP. Algorithm for the diagnosis and treatment of acute biliary pancreatitis. Scand J Surg. 2005;94(2):124-9.

21. Matull W'R. Pereira SP, O'Donohue JW. Biochemical markers of acute pancreatitis. J Clin Pathol. 2006 Apr;(4):340-4.

22. Kemppainen E, Puolakkainen P. Non-alcoholic etiologies of acute pancreatitisexclusion of other etiologic factors besides alcohol and gallstones. Pancreatology. 2007:(2-3):142-6.

23. Stimac D, Rubinic M, Lenac T, Kovac D, Vcev A. Miletic D. Biochemical parameters in the early differentiation of the etiology of acute pancreatitis. Am J Gastroenterol. 1996 Nov:91 (11):2355-9.

24. Folsch UR, Nitsche R, Ludtke R. Hilgers RA, Creutzfeldt W. Early ERCP and papillotomy compared with conservative treatment for acute biliary pancreatitis. The German Study Group on Acute Biliary Pancreatitis. N Engl J Med. 1997 Jan 23;(4):237-42.

25. Sanjay P. Yeeting S, Whigham C. Judson HK. Kulli C, Polignano FM, et al Management guidelines for gallstone pancreatitis.Are the targets achievable?. J Pancreas. 2009; (1):43-7.

26. Kimura Y, Takada T, Kawarada Y, Hirata K, Mayumi T, Yoshida M, et al. JPN Guidelines for the management of acute pancreatitis: treatment of gallstone-induced acute pancreatitis. J Hepatobiliary Pancreat Surg. 2006;(1):56-60.

27. Graham DF, Wyllie FJ. Prediction of gall-stone pancreatitis by computer. Br Med J. 1979 Feb 24;1(6162):515-7.

28. Campbell EJ, Montgomery DA, Mackay CJ. A national survey of current surgical treatment of acute gallstone disease. Surg Laparosc Endosc Percutan Tech. 2008 Jun;(3):242-7.

29. Kaw M. Al-Antably Y,Kaw P. Management of gallstone pancreatitis: cholecystectomy or ERCP and endoscopic sphincterotomy.Gastrointest Endosc. 2002 Jul;56(1):61-5. 